\title{
Visual Outcomes in Pediatric Patients with Peters
} Anomaly

\author{
Samiksha Fouzdar-Jain' \\ Zena Ibrahim ${ }^{2}$ \\ Jeremy Reitinger $\mathbb{1 D}^{3}$ \\ Dingcai $\mathrm{Cao}^{2}$ \\ Mehmet C Mocan ${ }^{2}$ \\ 'Department of Pediatric Ophthalmology \\ \& Adult Strabismus, Children's Hospital \\ and Medical Center, Omaha, NE, USA; \\ ${ }^{2}$ Department of Ophthalmology and \\ Visual Sciences, University of Illinois at \\ Chicago, Chicago, IL, USA; ${ }^{3}$ Creighton \\ University School of Medicine, Omaha, \\ NE, USA
}

Purpose: This study aimed to evaluate the visual outcomes of pediatric patients diagnosed with Peters anomaly (PA) in a tertiary eye care center.

Methods: This was a retrospective study undertaken at a single academic center. Clinical records of pediatric patients diagnosed with PA from 2000 to 2017 were reviewed. Parameters recorded included visual acuity (VA), intraocular pressure (IOP), presence of glaucoma, and surgical procedures performed. The impact of PA type on final visual acuity levels was analyzed using generalized estimation equation models.

Results: Twenty-one eyes from 11 patients were analyzed. At the final visit, 9 eyes $(42.8 \%)$ had a VA $\geq 20 / 200$, while $12(57 \%)$ had $<20 / 200$, ranging from $20 / 250$ to no light perception. Of the study cohort, 15 (71.5\%) eyes had type I, while six (28.5\%) had type II PA. Glaucoma was present in 20 eyes $(95 \%)$ at the final visit. One eye had dense central leucoma and microphthalmos where IOP could not be measured accurately. Seventeen eyes (81\%) underwent at least one intraocular surgery, while the average number of surgeries per eye was 2.9 \pm 2.5 (range $=0-9$ ). The type of PA beta(se) $=0.53(0.41), p=0.20]$ was not found to be a significant risk factor for worse VA.

Conclusion: Severe visual impairment develops in the majority of patients with PA. The type of anomaly does not appear to be associated with a worse visual function in patients with PA. Glaucoma develops in a high percentage of this patient population, and thus close monitoring of IOP in this patient population is crucial.

Keywords: Peters anomaly, penetrating keratoplasty, visual outcomes, logMAR, corneal opacity

\section{Introduction}

Peters anomaly (PA) is a rare congenital cause of anterior segment dysgenesis with central corneal opacification and a relatively clear peripheral cornea, with an incidence of approximately 1.5 per 100,000 births. $^{1,2}$ Type I PA is characterized by iridocorneal adhesions in addition to corneal opacification, whereas the more severe type II is characterized by additional keratolenticular adhesions with more extensive central corneal opacities. ${ }^{3}$

Due to the difference in the phenotypic presentation in patients with PA, as well as co-existing glaucoma in many of these patients, the visual prognosis in children with PA is highly variable; studies show anywhere from $10 \%$ to $54 \%$ of patients achieving visual acuity (VA) better than 20/200. ${ }^{4,5}$ Although variability in functional outcomes is often attributed to the severity of disease, the difference in keratoplasty techniques, frequency, and severity of graft rejection, and the presence of glaucoma has also been suggested as a potential confounder. ${ }^{4-6}$
Correspondence: Samiksha Fouzdar-jain Department of Pediatric Ophthalmology \& Adult Strabismus, Children's Hospital and Medical Center, Omaha, NE, USA Email sjain@childrensomaha.org 
The current study aimed to evaluate the visual function of pediatric patients with PA, report on the frequency of glaucoma in this patient population, and compare the visual outcomes based on the type of PA in a tertiary eye care center. We hypothesized that the type of PA might have additional comorbidity influencing the visual outcomes in patients with PA.

\section{Methods}

This retrospective study was undertaken at a single academic tertiary eye care center University of Illinois at Chicago, from January 2000 to December 2017. The study was approved by the Institutional Review Board (IRB\#20180491003) and adhered to the Declaration of Helsinki's tenets. It complied with the Health Insurance Portability and Accountability Act of 1996. Patient records were extracted using the institutional electronic medical record system. Informed consent for the subjects was waived due to the retrospective nature of the study. All the patients included in the study did not receive any ocular treatment before attending their first appointment within our clinic.

Clinical records of subjects reviewed included age at the initial visit and follow-up, duration of follow-up, VA, anterior segment findings, intraocular pressure (IOP), presence of glaucoma, and surgical procedures performed. The PA diagnosis was confirmed by identifying congenital corneal opacification with slit-lamp biomicroscopy and ultrasound biomicroscopy. Type I PA was diagnosed with the presence of central or paracentral corneal opacification with or without iridocorneal adhesions. Type II PA was diagnosed by the presence of corneal opacities and keratolenticular adhesions. Glaucoma was diagnosed when at least two IOP measurements above $21 \mathrm{mmHg}$ were noted together with evidence of glaucomatous optic neuropathy or corneal enlargement at any point during the patients' follow-up. Measurement of IOP was done at the time of examination under anesthesia using Tonopen XL (Reichert Instruments, Buffalo, NY). Patients who had visual function data and who were older than 3 years of age were included for data analysis at the final visit. Exclusion criteria included patients with undetermined corneal opacity type, Peters plus syndrome, less than six months of postoperative follow-up, less than one visit in total, no follow-up visit after three years of age (to exclude preverbal children), or incomplete clinical records.

Data were analyzed using Stata software (v15.1, StataCorp, College Station, Texas). VA data were converted to $\log$ MAR equivalents for statistical analysis, with "count fingers" and "hand motion" conversions according to previously established calculations by Schulze-Bonzel et al. $^{7}$ Generalized estimation equation models were used to assess the impact of the type of Peters anomaly on the final VA levels. This modeling allowed for between-eye correlations when both eyes of patients were included for analysis. Statistical significance for all tests was set at $p<0.05$.

\section{Results}

Twenty-nine eyes of 16 patients with an established diagnosis of PA were extracted from the electronic medical database. Out of this initial cohort, 21 eyes of 11 patients (6 male, 5 female) who were at least 3 years old at their final visit were included in the data analysis based on the inclusion criteria (Table 1). Of this cohort, ten patients had

Table I Demographic and Clinical Characteristics of Eyes with Type I versus Type II Peters Anomaly

\begin{tabular}{|c|c|c|c|}
\hline & $\begin{array}{l}\text { Total } \\
n=21 \\
\text { Eyes }\end{array}$ & $\begin{array}{c}\text { Type } \\
\text { I n=I } 5 \\
\text { Eyes }\end{array}$ & $\begin{array}{c}\text { Type II } \\
\text { n=6 Eyes }\end{array}$ \\
\hline $\begin{array}{l}\text { Final Visual Acuity } \\
\text { (logMAR) }\end{array}$ & $1.45 \pm 0.9$ & $1.27 \pm 0.8$ & $1.90 \pm 1.0$ \\
\hline \multicolumn{4}{|l|}{$\begin{array}{l}\text { Final Visual Acuity } \\
\text { (Snellen), N (\%) }\end{array}$} \\
\hline $\begin{array}{l}\text { Light Perception or } \\
\text { worse }\end{array}$ & $4(19 \%)$ & I (7\%) & $3(50 \%)$ \\
\hline Hand motion & I (5\%) & I (7\%) & $0(0 \%)$ \\
\hline Count Fingers & $3(14 \%)$ & $3(20 \%)$ & $0(0 \%)$ \\
\hline $20 / 400-20 / 200$ & $5(24 \%)$ & $3(20 \%)$ & $2(33 \%)$ \\
\hline $20 / 200-20 / 40$ & 7 (33\%) & $6(40 \%)$ & I (I7\%) \\
\hline Better than $20 / 40$ & I (5\%) & I (7\%) & $0(0 \%)$ \\
\hline Glaucoma, N (\%) & $20(95.2 \%)$ & 14 (93.3\%) & $6(100 \%)$ \\
\hline Initial IOP, $\mathrm{mmHg}$ & $22.2 \pm 7.1$ & $23.5 \pm 7.2$ & $19.0 \pm 6.3$ \\
\hline Final IOP, mmHg & $15.9 \pm 6.2$ & $15.5 \pm 6.7$ & $17.0 \pm 5.4$ \\
\hline Average surgeries per eye & $2.9 \pm 2.5$ & $3.0 \pm 2.6$ & $2.7 \pm 2.6$ \\
\hline $\begin{array}{l}\text { Frequency of Surgical } \\
\text { Intervention, } \mathrm{N}(\%)\end{array}$ & 17 (8I\%) & $12(80 \%)$ & $5(83 \%)$ \\
\hline Cataract & II (52\%) & $7(47 \%)$ & $4(66 \%)$ \\
\hline PKP/KPro & $8(38 \%)$ & $6(40 \%)$ & $2(33 \%)$ \\
\hline Glaucoma & $13(62 \%)$ & $10(67 \%)$ & $3(50 \%)$ \\
\hline $\begin{array}{l}\text { Both PKP/KPro and } \\
\text { Glaucoma }\end{array}$ & $6(29 \%)$ & $4(27 \%)$ & $2(33 \%)$ \\
\hline
\end{tabular}

Note: $P$ values denote statistical significance between parameters involving Type I and Type II Peters Anomaly eyes.

Abbreviations: PKP, penetrating keratoplasty; KPro, keratoprosthesis, IOP, intraocular Pressure. 
bilateral, and one patient had unilateral eye involvement. The mean presenting age of the 11 patients was $4.5 \pm 8.1$ months (range $=0.25-30$ months) while the final visit age was $9.1 \pm 3.7$ years (range $=61-151.75$ months). Mean follow-up was $94.5 \pm 9.8$ months (range $=48-149$ months).

Among the study eyes, 15 (71.4\%) eyes had Type I, and 6 (28.6\%) had Type II PA.

At the initial visit, 18 eyes had glaucoma, and three eyes of two patients did not have glaucoma. However, during their follow-up period, one patient who initially did not have glaucoma developed glaucoma in both eyes before final visual acuity levels were obtained.
Twenty (95.2\%) eyes had glaucoma, and one patient had dense central corneal opacity preventing any anterior or posterior segment examination and accurate measurement of IOP.

Nine (42.8\%) eyes had $>20 / 200$ VA, while $12(57 \%)$ eyes had VA worse than 20/200 (Figure 1). Among eyes with >VA 20/200, 7 eyes had Type I PA, and two eyes had Type II PA (Figure 2). Only one (4.8\%) eye had a VA of $\geq 20 / 40$. There was no significant difference between the final VA of eyes with type I versus Type II PA [ $1.27 \pm 0.80$ vs $1.90 \pm 1.03$, respectively; beta(se) $=0.53(0.41), p=0.20$ ] (Figure 3 ). The mean VA of

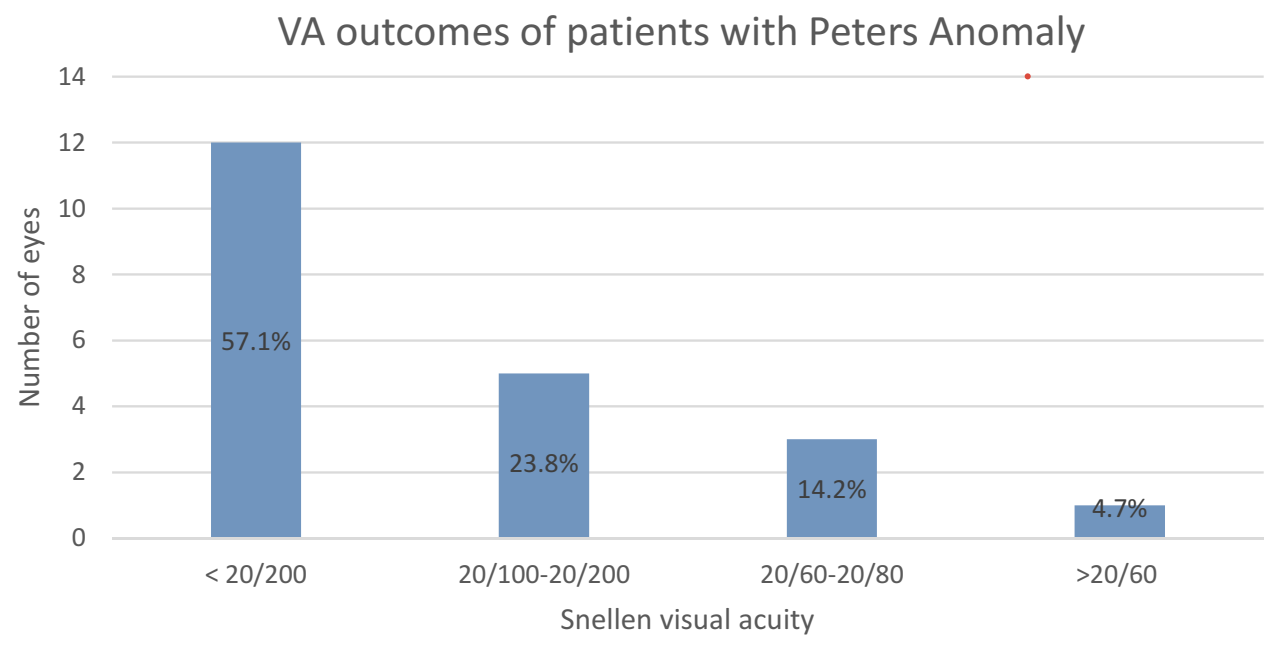

Figure I Stratification of visual acuity outcomes of study subjects with Peters anomaly.

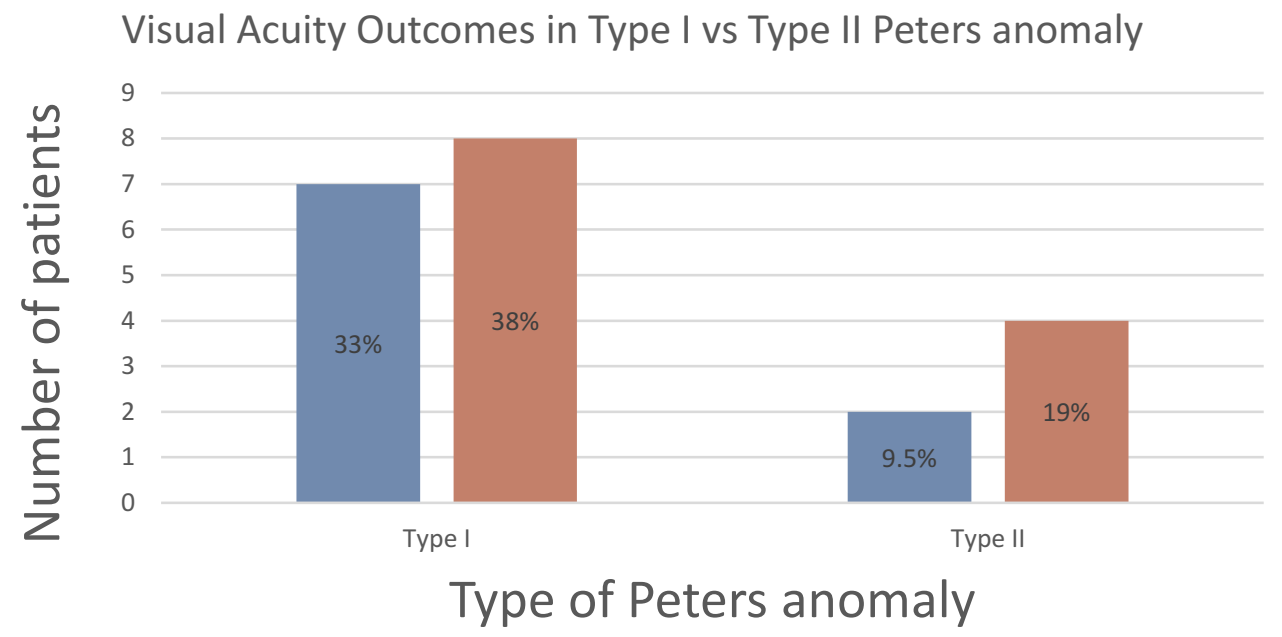

$\square \mathrm{VA} \geq 20 / 200 \square \mathrm{VA}<20 / 200$

Figure 2 Comparison of eyes with severe visual impairment $(\mathrm{VA}<20 / 200)$ in Type I versus Type II Peters anomaly. 


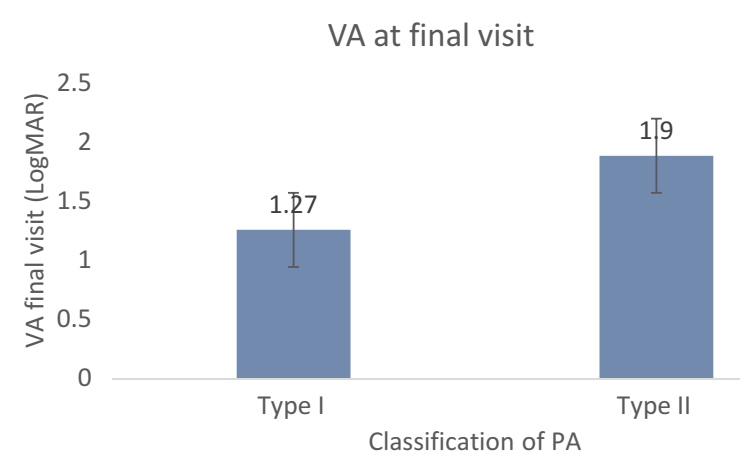

Figure 3 Comparison of mean visual acuity (VA) levels between type I and type II Peters Anomaly (PA).

the entire study cohort was determined as $1.45 \pm 0.89 \log$ MAR (range $=0.176-2.90$ ) Snellen equivalent 20/30 to No Light perception.

Seventeen eyes $(81 \%)$ received at least one surgery, while the average number of surgeries per eye was 2.9 \pm 2.5 . (range $=0-9$ ) (Table 1). The surgeries performed in the study eyes included cataract extraction, glaucoma drainage device implantation, penetrating keratoplasty, and sector iridectomy. In our study, only $38 \%$ of patients underwent keratoplasty, with a mean final VA of 1.29 $\pm 0.832 \log$ MAR range $=0.602-2.700$ (Snellen equivalent: 20/389.9) in 6 eyes with type 1 and 2.85 logMAR (Snellen equivalent:20/1588) in 2 eyes with type II PA. Six (40\%) eyes with type I and 2 (33\%) eyes with type II PA underwent keratoplasty. Authors are unable to comment at the age of corneal transplant surgery. Glaucoma was detected in $>90 \%$ of eyes in both groups (Table 1).

For all patients, the average IOP decreased from 22.2 $\mathrm{mmHg}$ at the initial visit to $15.8 \mathrm{mmHg}$ at the final visit [p<0.001] (Table 1). IOP measurements were obtained in the setting of variable degrees of corneal opacification, which may not reflect the true IOP levels in these abnormal eyes.

\section{Discussion}

Our results demonstrate that patients with PA end up with poor visual function and that this outcome does not appear to depend on the type of PA. We demonstrated that the majority of pediatric patients have final VA levels of $<20$ / 200, despite receiving appropriate surgical interventions and optical rehabilitation. Overall, our findings suggest that it is rare to obtain a VA of at least 20/40 or higher, affirming the outcomes of previous reports on this patient population. $^{4-6,8,9}$ In a cohort of 76 patients with differing severity of corneal opacification reported by Chang et al ${ }^{10}$ the average final VA of patients with PA was $2.049 \pm 0.965$ logMAR (20/2240). No difference was found between unilateral (2.05 $\pm 0.97 \mathrm{log}$ MAR) and bilateral $(2.05 \pm 0.97$ $\log$ MAR) cases, despite medical and/or surgical interventions. The results of this previous study are in agreement with those of ours, where we found a mean VA of 1.45 $\pm 0.89 \log$ MAR in our patients.

Although in our cohort, Type I PA patients appeared to have a better VA $(1.27 \pm 0.80 \log$ MAR [Snellen equivalent of 20/400]) compared to Type II patients $(1.90 \pm 1.00$ $\log$ MAR [counting fingers at approximately 1 foot]), this difference did not reach statistical significance $[p=0.20]$. To the best of our knowledge, although there has not been a direct comparison of VA outcomes in Type I and Type II PA patients, based on the results of previous studies, patients with type II tend to do end up with a worse visual function as compared to those with type I PA., ${ }^{5,11}$ In a study by Zaidman et $\mathrm{al}^{5}$ involving 16 cases with unilateral and 8 with bilateral type I PA, 54\% of eyes ended up with VA better than $20 / 100$ and $38 \%$ attained a final VA of $<20 / 200$. In a separate study by Yang et al, ${ }^{9} 75 \%$ of patients with type II PA ended with an eventual VA of LP or NLP. This difference is likely due to the higher prevalence of more severe corneal involvement, corneolenticular adhesions, or any associated ophthalmic anomalies such as cataracts or microphthalmia patients with type II PA.

Various studies have reported the presence of glaucoma in $50-70 \%$ of patients diagnosed with PA patients. ${ }^{1,3,12,13}$ However, the rate of glaucoma in our cohort was 95\%, much higher than reported in the ophthalmic literature. In addition, there was no difference in the rate of glaucoma between subjects with type I and II PA patients. It could be that the relatively longer follow-up time in our study $(94.5$ \pm 9.8 months) could have allowed for glaucoma to develop and thus be diagnosed in these anomalous eyes. We found that even in the presence of glaucoma, approximately $43 \%$ of patients achieved a VA of 20/200 or better. The findings of previous studies have suggested that glaucoma may negatively impact visual prognosis. ${ }^{4-6}$ Our study was not able to evaluate the impact of glaucoma on VA in patients with PA, as almost all of our study subjects had glaucoma as part of their ocular condition. However, it is possible that glaucoma may adversely affect visual outcomes, as shown in another study investigating the visual outcomes of patients with type I PA who underwent PKP. ${ }^{5}$ In that study, $75 \%$ of eyes without glaucoma had vision better 
than $20 / 100$, while only $36 \%$ of eyes with glaucoma were above this threshold. ${ }^{5}$

Glaucoma is challenging to treat in PA patients, with anywhere from $32 \%$ to $75 \%$ of eyes achieving sufficient IOP control. ${ }^{4,13}$ Dolezal et al have reported success following various glaucoma procedures, including glaucoma drainage devices (53\%), laser cycloablation (67\%) trabeculectomy with mitomycin $\mathrm{C}(25 \%)$ and trabeculotomy $(0 \%)$. They also found that $>50 \%$ of eyes with PA developed secondary glaucoma requiring multiple surgeries. ${ }^{4,13}$ However, good IOP control in our patients could be achieved in both Type I and II PA patients with medical and/or surgical interventions (Table 1).

Zaidman et $\mathrm{al}^{5}$ looked at visual outcomes after PKP in patients with type I PA, most of whom (97\%) received keratoplasty. In their older cohort, $66 \%$ of eyes had $\geq 20$ / $200 \mathrm{VA}$, and 7 out of 24 eyes (29\%) with type I PA had VA ranging from 20/20 to 20/50. In this group, nine of 12 (75\%) eyes without glaucoma and four of $11(36.4 \%)$ eyes with glaucoma had VA of $>20 / 100$. In our study, only $38 \%$ of patients underwent keratoplasty, with a mean final VA of $1.135 \log$ MAR (Snellen equivalent: 20/272) in 6 eyes with type 1 and $1.900 \operatorname{logMAR}$ (Snellen equivalent:20/ 1588 ) in 2 eyes with type II PA. The lower success rate in our cases could be related to the higher prevalence of glaucoma and the inclusion of patients with a more severe corneal opacification. In another study, Yang et $\mathrm{al}^{13}$ reported a final VA of $20 / 200$ or better in only $3(9 \%)$ eyes among 34 eyes of 19 children with PA, which reflects a poorer functional outcome than that $(9 / 21$ eyes; $42.8 \%)$ reported in our study. Visual outcomes after keratoplasty remain highly variable, and glaucoma development appears to be a poor prognostic indicator, as suggested by previous studies. ${ }^{8,9,14}$ (Table 1 ).

The limitations of our study include the small sample size and its retrospective nature. Due to the limited sample size, the presence of several potentially confounding factors such as anterior segment eye anomalies and the type of procedures performed could not be accounted for in the statistical analysis of the patients due to the high prevalence of glaucoma (95\%) in our study, we were unable to compare the outcomes of eyes with and without glaucoma. Another limitation is that glaucoma severity was not assessed in our study. Patients with end-stage glaucoma will likely have a marked reduction in VA due to optic neuropathy compared to those at the early stages of the disease. The study's retrospective nature made the collection of pertinent data, such as VA and IOP difficult. For several patients, visual function was not recorded in Snellen notation. On the other hand, one strength of our study is the inclusion of severely affected patients with PA. Thus, our results complement the outcomes of other studies that previously investigated PA outcomes with less severe involvement or those with only Type I PA.

In conclusion, our results suggest that patients with type I and type II PA have similar visual outcomes, and ambulatory vision may be attained in at least a minority of patients with PA. Although treatment outcomes continue to be highly variable, the visual outcomes of patients with PA continue to be dismal, with only $5 \%$ of PA eyes attaining a VA of better than $20 / 40$. It is imperative to manage these patients with a multidisciplinary approach, including prompt and aggressive treatment of amblyopia secondary to deprivation, which is necessary for better clinical outcomes.

\section{Acknowledgment}

This paper's abstract was presented at the AAPOS Annual Meeting 2019 as a poster presentation with interim findings.

\section{Funding}

This study was supported by a core grant from NEI (P30 EY001792, Core Grant for Vision Research) and an unrestricted grant from Research to Prevent Blindness.

\section{Disclosure}

The authors report no conflicts of interest.

\section{References}

1. Shigeyasu C, Yamada M, Mizuno Y, Yokoi T, Nishina S, Azuma N. Clinical features of anterior segment dysgenesis associated with congenital corneal opacities. Cornea. 2012;31(3):293-298. doi:10.1097/ ICO.0b013e31820cd2ab

2. Kurilec JM, Zaidman GW. Incidence of Peters anomaly and congenital corneal opacities interfering with vision in the United States. Cornea. 2014;33(8):848-850. doi:10.1097/ICO.0000000000000182

3. Bhandari R, Ferri S, Whittaker B, Liu M, Lazzaro DR. Peters anomaly: review of the literature. Cornea. 2011;30(8):939-944. doi:10.10 97/ICO.0b013e31820156a9

4. Dolezal KA, Besirli CG, Mian SI, Sugar A, Moroi SE, Bohnsack BL. Glaucoma and cornea surgery outcomes in Peters anomaly. Am J Ophthalmol. 2019;208:367-375. doi:10.1016/j.ajo.2019.08.012

5. Zaidman GW, Flanagan JK, Furey CC. Long-term visual prognosis in children after corneal transplant surgery for Peters anomaly type I. Am J Ophthalmol. 2007;144(1):104-108. doi:10.1016/j.ajo.2007.03.058

6. Gollamudi SR, Traboulsi EI, Chamon W, Stark WJ, Maumenee IH. Visual outcome after surgery for Peters' anomaly. Ophthalmic Genet. 1994;15(1):31-35. doi:10.3109/13816819409056908

7. Schulze-Bonsel K, Feltgen N, Burau H, Hansen L, Bach M. Visual acuities "hand motion" and "counting fingers" can be quantified with the Freiburg visual acuity test. Investig Opthalmol Visual Sci. 2006;47 (3):1236. doi:10.1167/iovs.05-0981 
8. Chang JW, Kim MK, Kim JH, Kim SJ, Wee WR, Yu YS. Long-term visual outcomes of penetrating keratoplasty for Peters anomaly. Graefes Arch Clin Exp Ophthalmol. 2013;251(3):953-958. doi:10. 1007/s00417-012-2165-7

9. Yang LL, Lambert SR, Drews-Botsch C, Stulting RD. Long-term visual outcome of penetrating keratoplasty in infants and children with Peters anomaly. J AAPOS. 2009;13(2):175-180. doi:10.1016/j. jaapos.2008.10.007

10. Chang JW, Kim JH, Kim SJ, Yu YS. Long-term clinical course and visual outcome associated with Peters' anomaly. Eye (Lond). 2012;26 (9):1237-1242. doi:10.1038/eye.2012.128

11. Elbaz U, Strungaru H, Mireskandari K, Stephens D, Ali A. Longterm visual outcomes and clinical course of patients with Peters anomaly. Cornea. 2020. doi:10.1097/ICO.0000000000002577
12. Stone DL, Kenyon KR, Green WR, Ryan SJ. Congenital central corneal leukoma (Peters' anomaly). Am J Ophthalmol. 1976;81 (2):173-193. doi:10.1016/0002-9394(76)90729-7

13. Yang LLH, Lambert SR, Lynn MJ, Stulting RD. Surgical management of glaucoma in infants and children with Peters anomaly. Ophthalmology. 2004;111(1):112-117. doi:10.1016/j.ophtha.2003.02. 002

14. Yoshikawa H, Sotozono C, Ikeda Y, Mori K, Ueno M, Kinoshita S. Long-term clinical course in eyes with Peters anomaly. Cornea. 2017;36(4):448-451. doi:10.1097/ICO.0000000000001161
Clinical Ophthalmology

\section{Publish your work in this journal}

Clinical Ophthalmology is an international, peer-reviewed journal covering all subspecialties within ophthalmology. Key topics include: Optometry; Visual science; Pharmacology and drug therapy in eye diseases; Basic Sciences; Primary and Secondary eye care; Patient Safety and Quality of Care Improvements. This journal is indexed on PubMed

Submit your manuscript here: https://www.dovepress.com/clinical-ophthalmology-journal

\section{Dovepress}

Central and CAS, and is the official journal of The Society of Clinical Ophthalmology (SCO). The manuscript management system is completely online and includes a very quick and fair peer-review system, which is all easy to use. Visit http://www.dovepress.com/ testimonials.php to read real quotes from published authors. 